\title{
AVALIAÇÃO FARMACOECONÔMICA DE DIFERENTES MARCAS DE DIMETICO- NA COMERCIALIZADA EM ANÁPOLIS - GO
}

\author{
PHARMACOECONOMICS ASSESSMENT OF DIFERENT BRANDS OF DIMETI- \\ CONA MARKETED IN ANÁPOLIS - GO
}

\author{
ARRUDA, Marllon Christian; OLIVEIRA, Tiago Branquinho² \\ ${ }^{1}$ Graduando do curso de Farmácia, Centro Universitário de Anápolis - UniEVANGÉLICA. Rua Dr. Evandro P. \\ Silva Qd 1 Lt 9/10 Bairro Cidade Universitária: marllon_enzo@hotmail.com \\ ${ }^{2}$ Mestre em Ciências Farmacêuticas, Orientador, Centro Universitário de Anápolis - UniEVANGÉLICA. Avenida \\ Universitária Km 3,5 Cidade Universitária 75070-290 - Anápolis, GO - Brasil: tiago.oliveira@unievangelica.edu.br
}

\section{RESUMO}

A farmacoeconomia estuda a relação entre opções de tratamento, avaliando o custobenefício. Os farmacêuticos, médicos e outros profissionais da área da saúde estão procurando selecionar métodos adequados para efetivar seus programas de saúde e para prescrever medicamentos aos seus pacientes devido às mudanças na estrutura econômica do sistema de saúde. O tamanho da gota pode ser influenciado por vários fatores como densidade do líquido, temperatura, tensão superficial, viscosidade, diâmetro e abertura da extremidade do gotejador e do ângulo de gotejamento. $\mathrm{O}$ objetivo deste trabalho foi identificar, medir e comparar os custos da utilização da dimeticona na forma farmacêutica de gotas. Realizou-se um estudo experimental utilizando frascos, de cinco marcas diferentes de Dimeticona em gotas comercializadas no mercado de Anápolis GO. Observou-se que o volume da gota variou de $0,0286 \mathrm{~mL}$ a $0,0746 \mathrm{~mL}$, o número de gotas variou conforme o volume dos frascos: 486 a 524 gotas em frascos de $15 \mathrm{~mL}$ e 191 a 243 gotas em frascos de $10 \mathrm{~mL}$, o custo da gota teve uma variação de 184,21\%, não houve variação significativa entre a densidade dos cinco medicamentos. Apenas dois medicamentos (um genérico e um similar) continham o volume dentro do valor especificado pelos fabricantes. Conclui-se que os medicamentos genéricos foram os que tiveram o menor custo durante o tratamento, por outro lado os medicamentos similares foram os de maior custo contrastando com o menor valor unitário.

Palavras-chave: Farmacoeconomia. Dimeticona. Gotas.

\begin{abstract}
The Pharmacoeconomics study the relation among treatment options, valuing the benefit cost. The pharmaceutical, doctors and others health professional area trying to select rights methods to became permanent your healf programs and to prescribe medicines to your patients due the chenges on the economical structure of health sistem. The proposeof this assigment was identify, measure and to compare the dimeticona utility on drops pharmaceutical form through the drop size to be influenced for several fators as liquid dencity, temperature, superfical tension,texture, diameter and opennig dropper and dropper angle. Has realizede an experimental study using flasks of five diferents brands of Dimeticona drops marketed in Anapolis-Go. Has noticed that the drops volume has changed $0,0286 \mathrm{~mL}$ until $0,0746 \mathrm{~mL}$, the drops number has chenged according the flasks volume: 486 until 524 drops flasks of $15 \mathrm{ml}$ and 191 until 243 drops flasks of $10 \mathrm{~mL}$, the drops cost had changed $184,21 \%$ there wasn't mean change among the density of the five medicines. Only two medicines (one generic and one similar) conteined the volume into the value discriminated by makers. To conclude tat generic medicines have had the less cost
\end{abstract}


during the treatment, on other side the similares medicines were the ones of the higther cost contresting with less value unit.

Key-words: Pharmacoeconomics. Dimeticona. Drops

\section{INTRODUÇÃO}

O desenvolvimento histórico da farmacoeconomia pode ser traçado através das ciências econômicas. Economia da saúde, uma área aplicada da economia concebida nos anos 1960, direciona especificamente como as ferramentas econômicas podem ser usadas para analisar a saúde e os problemas de assistência a saúde. Na década de 90 , o aumento dos custos a assistência à saúde e o reconhecimento dos recursos finitos nessa área cria uma pressão para determinar o valor de cada gasto com a saúde, este fato motivou a farmacoeconomia a sair da condição de ciência teórica para tornar-se uma ciência prática e resultou na inclusão na grade curricular de muitos cursos em graduação em farmácia (BISSON, 2007).

Nos países em desenvolvimento, como o Brasil, a Farmacoeconomia deve incluir, além da avaliação econômica de medicamentos, outros enfoques de importância na garantia do acesso e uso racional dos medicamentos (MOTA et al., 2003).

A avaliação farmacoeconômica consiste em um conjunto de procedimentos ou técnicas relacionadas à identificação, medição e quantificação dos efeitos sobre a saúde e sobre os recursos econômicos, permitindo eleger entre duas ou mais alternativas terapêuticas a que melhor resultado origina para a sociedade. As publicações internacionais têm evidenciado o uso crescente das técnicas de avaliação econômica para subsidiar a tomada de decisões na gestão sanitária. Os estudos de avaliação farmacoeconômica medem e comparam alternativas farmacológicas/ farmacêuticas em função de seus custos e benefícios, com o intuito de selecionar a mais eficiente para a sociedade (GUIMARÃES et al., 2007; MOTA et al., 2003).

Os farmacêuticos, médicos e outros profissionais da área da saúde estão procurando selecionar métodos adequados para efetivar seus programas de saúde e para prescrever medicamentos aos seus pacientes devido às mudanças na estrutura econômica do sistema de saúde. Desta forma, a farmacoeconomia está sendo uma ferramenta bastante utilizada para proporcionar aos pacientes benefícios nos resultados clínicos e econômicos, praticando análises como a de minimização, que tem por finalidade reduzir os custos e comparar as opções de medicamentos e tratamentos disponíveis, buscando, entre as equivalentes, a de menor custo (CYRILLO, 2004; SECOLI et al., 2005).

Os gastos com saúde vêm crescendo em ritmo acelerado em âmbito mundial, preocupando usuários, governos e sociedade desta forma a farmacoeconomia objetiva a otimização do uso de recursos financeiros sem que ocorra prejuízo na qualidade do tratamento oferecido identificando, calculando e comparando custos (recursos consumidos), riscos e benefícios (clínicos, econômicos, humanísticos) de programas ou terapias específicas, e determinando quais são as alternativas que produzem os melhores resultados em face dos recursos investidos (DALTIO et al., 2007; GUIMARÃES et al., 2007; SECOLI et al., 2005).

A dimeticona é um óleo inerte de silicone com propriedades de aderir-se à pele e 
ser repelente à água. Tem ainda propriedades protetoras sobre o trato gastrointestinal e ação adsorvente. É considerada como adjuvante no tratamento de condições em que o gás localizado no trato intestinal é um problema, como na flatulência, distensão gasosa pós-cirúrgica e para eliminar gases que possam dificultar a interpretação de exames radiográficos abdominais. É reconhecida como sendo fisiologicamente inerte e sem toxicidade por não ser absorvida pela mucosa gastrointestinal, sendo utilizada somente por via oral, na forma de solução ou comprimido (GOODMAN, 2003; KOROLKOVAS; FRANÇA, 2006/07).

A dimeticona atua no estômago e no intestino, diminuindo a tensão superficial dos líquidos digestivos, rompendo ou dificultando a formação de bolhas que retêm os gases e que são responsáveis pela dor abdominal e flatulência resultando no alívio dos sintomas associados com a retenção de gases. Ela é indicada para pacientes com excessos de gases no aparelho digestivo (BRISTOL-MYERS, 2008).

O tamanho da gota é influenciado por vários fatores como densidade do líquido, temperatura, tensão superficial, viscosidade, diâmetro e abertura da extremidade do gotejador e do ângulo de gotejamento (FERREIRA, 2007). Por isso a produção desta forma farmacêutica é considerada um processo de difícil uniformização, sendo evitadas prescrições em gota quando se deseja uma dose exata de um medicamento (JOAQUÍN, 1999).

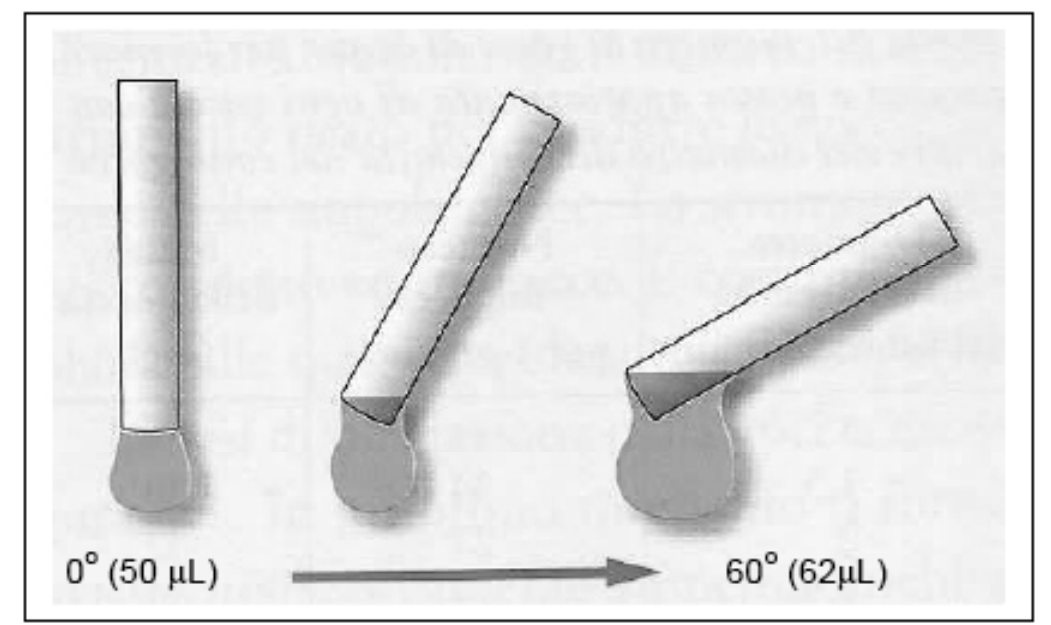

FIGURA 1: AUMENTO PROGRESSIVO DO VOLUME DA GOTA DE ÁGUA COM AUMENTO DA ÂNGULO DE INCLINAÇÃO DO CONTA-GOTAS (CONTA-GOTAS OFICIAL $\left.25^{\circ} \mathrm{C}\right)$. FONTE: FERREIRA, 2007.

A forma farmacêutica "gotas" é frequentemente empregada como unidade de medida de um medicamento. Entretanto, como medida, a gota por si só não representa uma massa ou volume específico, uma vez que o volume das gotas de diferentes líquidos varia muito entre si (ANSEL; STOKLOSA, 2001).

O presente estudo propõe a investigação em farmacoeconomia buscando identificar, medir e comparar os custos da utilização dos medicamentos, e assim, avaliar o volume do frasco dos medicamentos, verificar a densidade dos medicamentos, 
determinar o volume e o peso da gota, avaliar o custo da gota, avaliar o custo do tratamento máximo diário e comparar o custo entre os medicamentos (referência, genérico, similar).

\section{MATERIAL E MÉTODOS}

Realizou-se um estudo experimental utilizando frascos, de cinco marcas diferentes de Dimeticona em gotas, dentre elas, uma marca referência, duas marcas similares e duas marcas genéricas: Luftal, For Gas, Neo Dimeticon, Dimeticona EMS, Dimeticona - Medley, respectivamente. Este estudo adaptou a metodologia de Alcantara, Virtuoso e Oliveira (2007); Roizenblatt et al. (2001); Stillitano et al. (2003); Prata Júnior e Prata (2004).

Comparou-se o peso de cada gota em balança analítica Gehaka modelo AG200 Classe 1, devidamente calibrada (conforme protocolo 08102201001015), em temperatura ambiente de $21^{\circ} \mathrm{C}$.

Para obtenção da gota o frasco de dimeticona foi invertido a $90^{\circ}$, e levemente pressionado a fim de proporcionar uma gota após aproximadamente 10 segundos. Todos os frascos de dimeticona foram abertos e testados pelo mesmo investigador e foram desprezadas dez gotas de cada frasco, em triplicata, foram pesadas dentro de um becker previamente tarado para estabelecer a medida referente a cada amostra. A balança foi tarada a cada medida (Figura 2-A).

$O$ restante do medicamento contido no frasco foi pesado e somando com a medida já calculada de cada frasco (10 gotas), calculou-se o volume total dos frascos de dimeticona (Figura 2-B) após a determinação do volume da gota, a qual foi obtida após avaliação da média de densidade de cada medicamento avaliado conforme a Farmacopéia Brasileira IV (1988) (Figura 2-C).
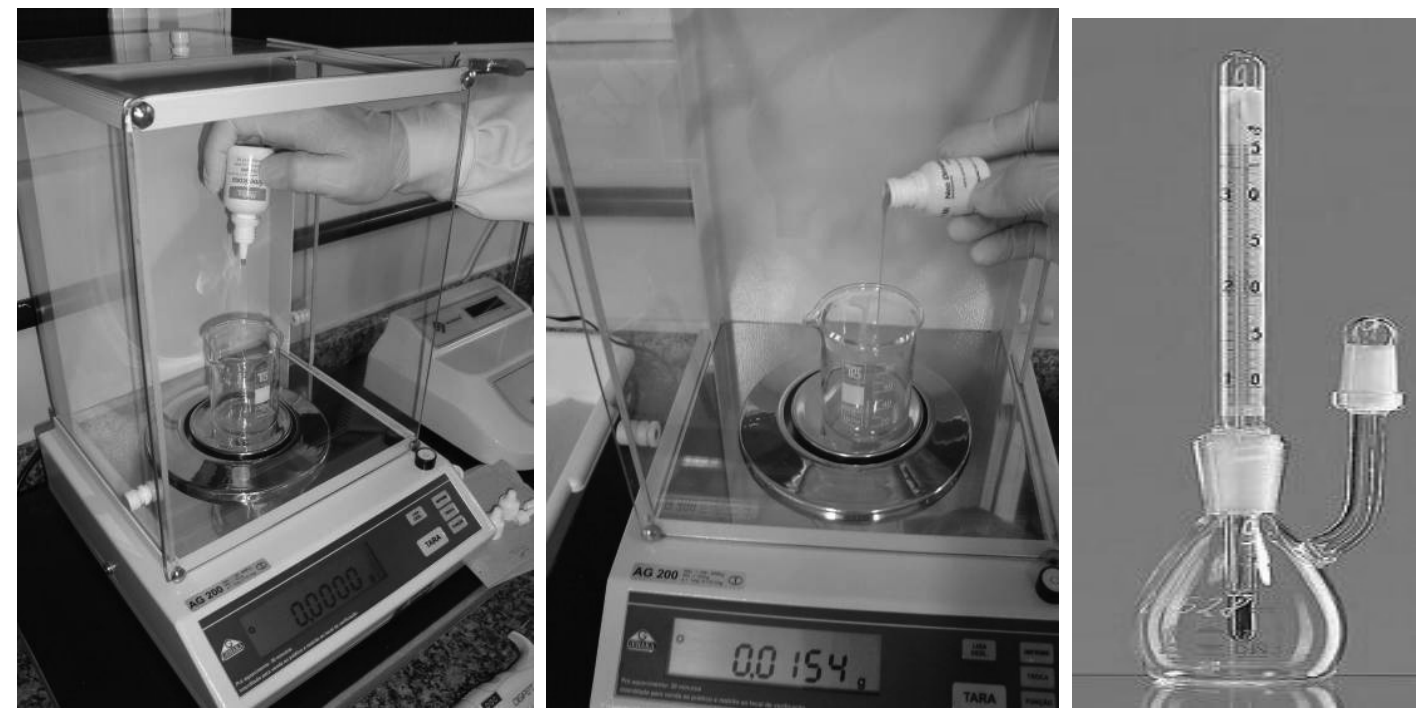

FIGURA 2: SEQÜÊNCIA DE PESAGEM: A - PESAGEM DAS GOTAS; B - PESAGEM TOTAL DO MEDICAMENTO; C - DENSIDADE DO MEDICAMENTO. RESULTADO DO PRÓPRIO AUTOR. 
Através das medidas obtidas na determinação do volume das gotas, número de gotas e volume total em cada um dos frascos, foi possível calcular o custo diário do tratamento para cada um dos medicamentos, considerando a posologia máxima para cada tipo de tratamento ( 6 gotas para lactentes, 12 gotas para crianças e 16 gotas para adultos, com 3 administrações diárias), a duração para cada tipo de tratamento (4 dias) e os preços das medicações conforme a Câmara de Regulação do Mercado de Medicamentos.

\section{RESULTADOS E DISCUSSÃO}

Foram utilizados na pesquisa os medicamentos: Luftal (referência), Dimeticona - EMS e Dimeticona - Medley (genéricas) na apresentação de gotas em frascos de $15 \mathrm{~mL}$. For Gas e Neo Dimeticon (similares) em frasco de $10 \mathrm{~mL}$. O volume da gota variou de $0,0286 \mathrm{~mL}$ a $0,0746 \mathrm{~mL}$, o número de gotas variou conforme o volume dos frascos: 486 a 524 gotas em frascos de $15 \mathrm{~mL}$ e 191 a 243 gotas em frascos de 10 $\mathrm{mL}$, o custo da gota teve uma variação de $184,21 \%$ e a densidade encontrada em cada medicamento foram: Luftal - 1,0150 g/mL; Dimeticona - EMS - 1,0147 g/mL; Dimeticona - Medley - 1,0136 g/mL; For Gas - 1,0148 g/mL; Neo Dimeticon - 1,0141 $\mathrm{g} / \mathrm{mL}$ (Tabela 1).

TABELA 1: AVALIAÇÃO FARMACOECONÔMICA DE 5 MARCAS DE DIMETICONA

\begin{tabular}{|c|c|c|c|c|c|c|c|}
\hline Amostra & $\begin{array}{c}\text { Volume } \\
\text { total } \\
(\mathrm{mL})\end{array}$ & $\begin{array}{c}\text { Volume } \\
\text { da gota } \\
\text { (mL) }\end{array}$ & $\begin{array}{l}\text { Desvio } \\
\text { padrão }\end{array}$ & $\begin{array}{l}N^{0} \text { de } \\
\text { gotas }\end{array}$ & $\begin{array}{c}\text { Preço } \\
\text { do } \\
\text { fras- } \\
\text { co } \\
(R \$)\end{array}$ & $\begin{array}{l}\text { Custo } \\
\text { da } \\
\text { gota } \\
\text { (R\$) }\end{array}$ & $\begin{array}{c}\text { Densidade } \\
(\mathrm{g} / \mathrm{mL})\end{array}$ \\
\hline Luftal & 14,4229 & 0,0298 & 0,0012 & 486 & 13,20 & 0,027 & 1,0150 \\
\hline Dimet. EMS & 14,0748 & 0,0286 & 0,0007 & 490 & 9,84 & 0,020 & 1,0147 \\
\hline Dimet. Medley & 15,4348 & 0,0295 & 0,0014 & 524 & 10,18 & 0,019 & 1,0136 \\
\hline For Gas & 10,0603 & 0,0411 & 0,0033 & 243 & 8,34 & 0,034 & 1,0148 \\
\hline Neo Dimeticon & 9,8478 & 0,0746 & 0,0046 & 191 & 6,86 & 0,035 & 1,0141 \\
\hline
\end{tabular}

Fonte: Resultado do próprio autor.

Observou-se que apenas um medicamento genérico e um similar continham o volume dentro do valor especificado pelos fabricantes $(15,4348 \mathrm{~mL}$ e $10,0603 \mathrm{~mL}$ respectivamente), enquanto que os demais medicamentos apresentaram volumes abaixo do estabelecido, sendo que a Dimeticona - EMS possuía o menor volume $(14,0748 \mathrm{~mL})$, tal fato representa uma lesão aos Direitos do Consumidor uma vez que a quantidade líquida de uma embalagem não deve ser menor que o especificado em sua rotulagem, pois caso isso ocorra o consumidor pode exigir o abatimento proporcional do preço, complementação do peso ou medida, a substituição do produto por outro da mesma espécie, marca ou modelo, sem os aludidos vícios ou restituição imediata da quantia paga, monetariamente atualizada, sem prejuízo de eventuais perdas e 
danos (BRASIL, 1990). Essas variações não demandam apenas em aspecto ético, pois é um modo que o fabricante tem para aumentar sua lucratividade, mas também problemas de Boas Práticas de Fabricação (BPF), pois as mesmas asseguram que os produtos são consistentemente produzidos e controlados, com padrões de qualidade apropriados para o uso pretendido e requerido pelo registro (BRASIL/ AGÊNCIA NACIONAL DE VIGILÂNCIA SANITÁRIA, 2003).

Houve também uma variação entre a média do volume da gota de cada medicamento, onde a Dimeticona - EMS apresentou o menor volume $(0,0286 \mathrm{~mL})$ e o Neo Dimeticon o maior volume $(0,0746 \mathrm{~mL})$, isso resulta de variações da viscosidade da solução, da abertura do orifício do gotejador e até da temperatura ambiente, podendo gerar uma concentração menor do fármaco por dose, caracterizando um possível problema de subdose na utilização do medicamento (ALCANTARA; VIRTUOSO; OLIVEIRA, 2007).

Variações amplas no número de gotas por frascos de drogas diferentes, mas com mesmo volume têm sido reportadas em outros estudos, assim como a contagem do número de gotas entre vários frascos de um mesmo produto (STILLITANO et al., 2003).

Considerando o preço unitário de cada medicamento e seu número de gotas, calculou-se o custo da gota de cada medicamento (Gráfico 1) que apresentou o Neo Dimeticon como o medicamento que possui a gota com o valor mais alto $(R \$ 0,035)$ e a Dimeticona - Medley com o valor mais baixo $(R \$ 0,019)$.

\section{GRÁFICO 1: CUSTO DA GOTA DOS MEDICAMENTOS.}

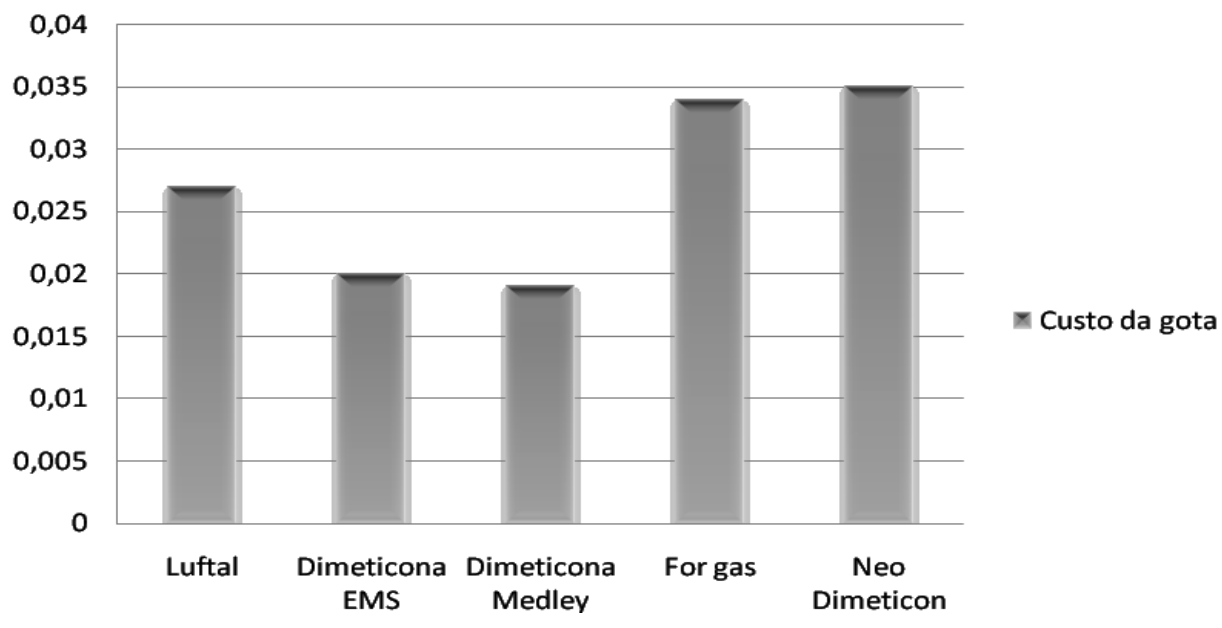

FONTE: RESULTADO DO PRÓPRIO AUTOR

Segundo Joaquín (1999), uma variação aceitável no volume das gotas/conteúdo de medicamento fornecido a crianças situa-se em torno de $10 \%$ da dose estimada, levando-se em conta estas variações.

Segundo Alcantara (2007), verificou-se que, a densidade do produto não é compatível com o orifício do gotejador. Para solucionar este problema, as formulações 
de menor densidade teriam que ter o orifício do gotejador diminuído, e as de maior densidade, aumentado. Outra opção seria modificar a densidade da formulação, para ser compatível com o tamanho do orifício.

$\mathrm{Na}$ comparação do custo (em reais) do tratamento em gotas de dimeticona, considerando o máximo de gotas que podem ser administradas diariamente e em 4 dias por adultos, crianças e lactentes (Gráficos 2 e 3). O custo do tratamento adulto se apresentou mais elevado que o lactente e pediátrico, em todas as amostras, devido diretamente à posologia para cada tipo de tratamento, uma vez que o tratamento máximo para lactentes 72 gotas, crianças 144 gotas e adultos exige 192 gotas (GOODMAN, 2003). Os dados encontrados possibilitaram a observação da diferença entre o preço do tratamento por crise (cólicas) com medicamentos de referência, genéricos e similares em indivíduos lactentes, crianças e adultos (Gráfico 2). Verificando que o tratamento com medicamento genérico foi o que teve o menor custo (Dimeticona EMS e Dimeticona - Medley) observando que nesta classe de medicamento a entrada dos genéricos no mercado incentivou a concorrência no setor, trazendo novas opções ao consumidor o que provavelmente e fez cair os preços (MINISTÉRIO DA SAÚDE/ AGÊNCIA NACIONAL DE VIGILÂNCIA SANITÁRIA, 2002). Os medicamentos similares foram o que apresentaram maior custo para o tratamento (For Gas e Neo Dimeticon). A posologia encontrada na bula de cinco (100\%) amostras foi a mesma da literatura.

\section{GRÁFICO 2: CUSTO DO TRATAMENTO DIÁRIO (R\$).}

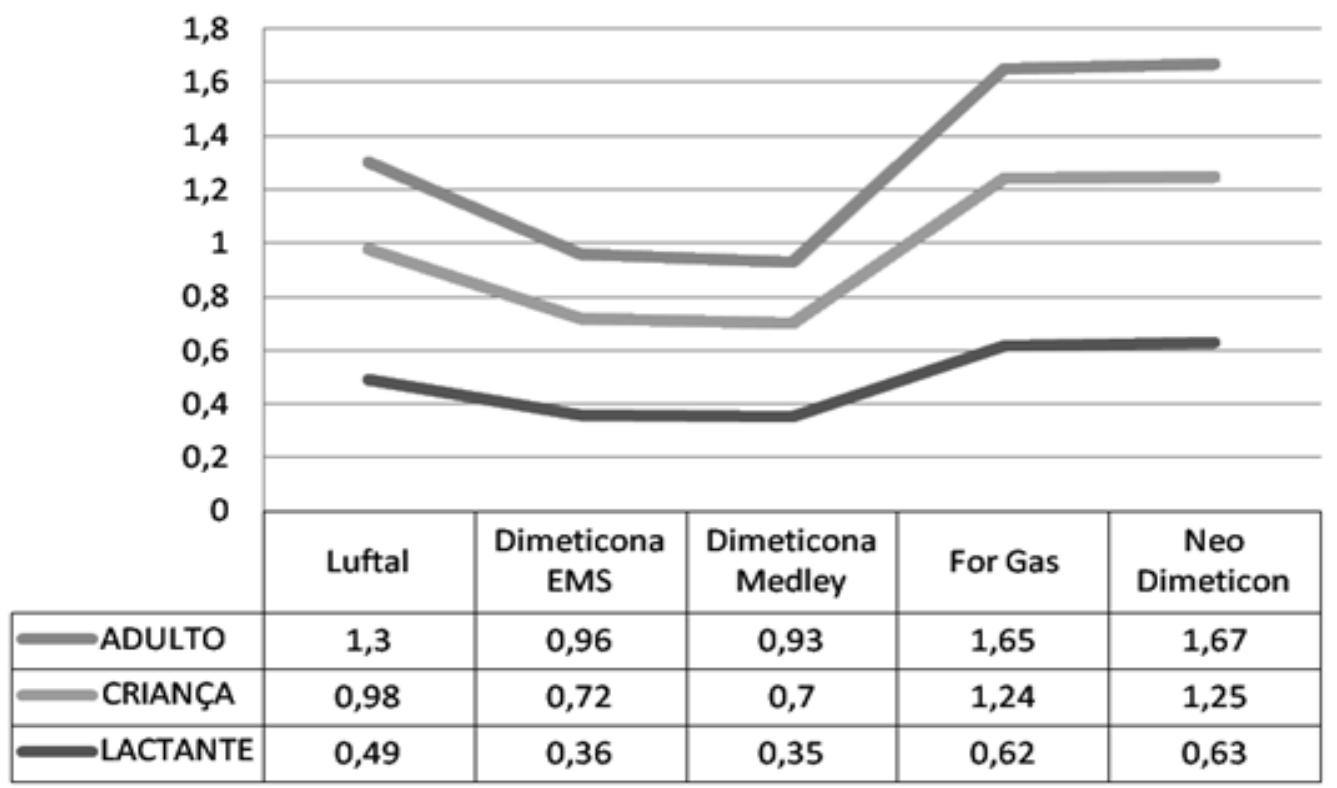

FONTE: RESULTADO DO PRÓPRIO AUTOR 


\section{GRÁFICO 3: CUSTO DO TRATAMENTO EM REAIS POR CRISES (CÓLICAS).}

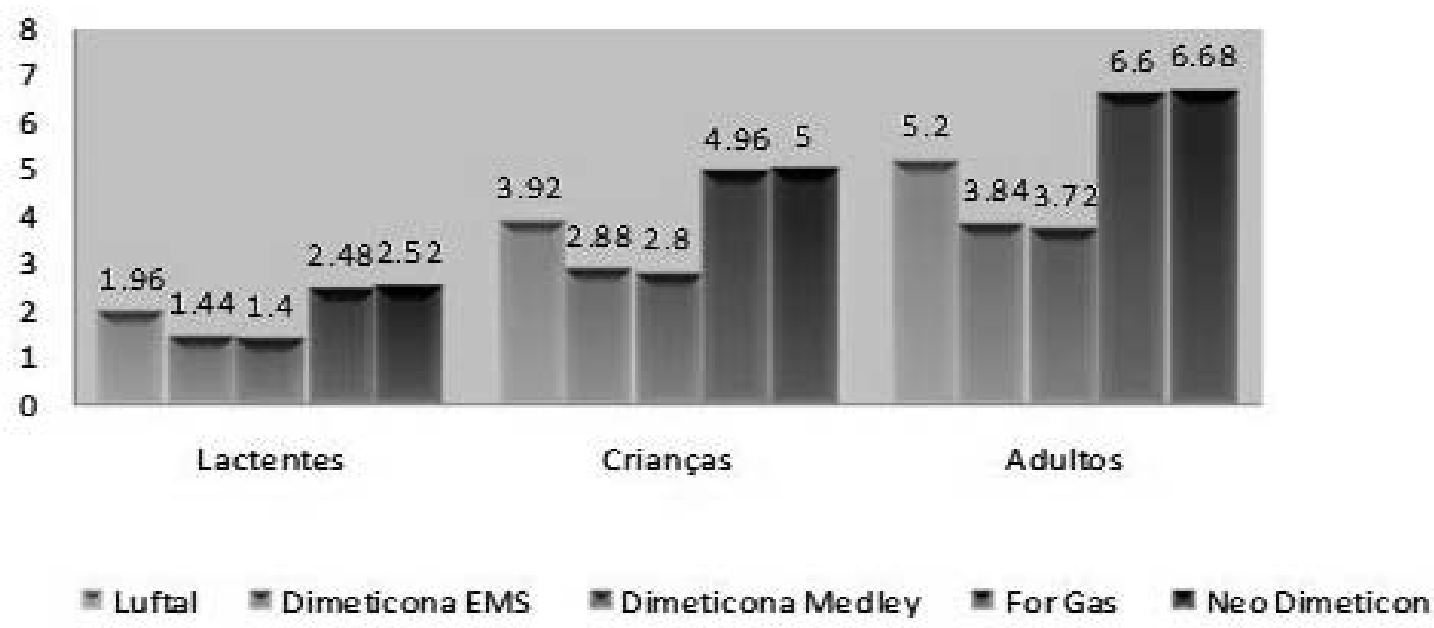

FONTE: RESULTADO DO PRÓPRIO AUTOR

\section{CONCLUSÃO}

Por meio deste estudo observou-se que somente dois medicamentos tiveram a quantidade indicada no rótulo. A densidade não teve variação significativa $(1,0136$ a $1,0150 \mathrm{~g} / \mathrm{mL}$ ). $O$ volume da gota encontrado teve uma diferença significativa. $O$ custo da gota teve uma variação de $184,21 \%$, sendo que a Dimeticona Medley foi a que apresentou o menor custo da gota $(R \$ 0,019)$ enquanto o Neo Dimeticon foi o que apresentou o maior custo da gota $(R \$ 0,035)$. Os medicamentos genéricos (Dimeticona - EMS e Dimeticona - Medley) foram os que tiveram o menor custo durante o tratamento, por outro lado os medicamentos similares (For Gas e Neo Dimeticon) foram os de maior custo contrastando com o menor valor unitário.

Os dados do presente estudo dão suporte à hipótese que existem diferenças no tratamento com o medicamento dimeticona gotas o que deve ser considerado no processo decisório de compras.

\section{REFERÊNCIAS}

ANSEL, H.C. \& STOKLOSA, M.J. Pharmaceutical Calculations. 11th ed. Philadelphia: Lippincott Williams \& Wilkins, 2001. p.66-67.

BISSON, Marcelo Polacow. Farmácia clínica \& atenção farmacêutica. $2^{\mathrm{a}}$ ed. Barueri: Manole, 2007. 
BRASIL/ AGÊNCIA NACIONAL DE VIGILÂNCIA SANITÁRIA. Medicamentos Genéricos: oriente-se. Brasília: ANVISA, 2002.

BRASIL/ AGÊNCIA NACIONAL DE VIGILÂNCIA SANITÁRIA. RDC n 210 , de de 04 de agosto de 2003. Diário Oficial da União; Poder Executivo, Brasília, DF, 14 ago. 2003. Seção 3. On line. Disponível em:http://www.anvisa.gov.br/legis/resol/ 2003/ rdc/210_03rdc.pdf. Acesso em: 05 de outubro de 2009.

BRASIL. LEI No 8.078, DE 11 DE SETEMBRO DE 1990. Disponível em: https://www. planalto.gov.br/ccivil_03/leis//8078.htm Acesso em 28 de maio de 2009.

BRISTOL-MYERS SQUIBB. Bula de medicamento: Dimeticona gotas (Luftal). São Paulo, 2008.

CYRILLO, Marcos Antônio. Farmacoeconomia e suas aplicações. Prática Hospitalar, ano X, no. 58, jul.-ago. 2004.

DALTIO, Claudiane Salles; MARI, Jair de Jesus; FERRAZ, Marcos Bosi. Estudos farmacoeconômicos e carga da doença em esquizofrenia. Revista de psiquiatria clínica. 2007, vol.34, suppl.2, pp. 208-212.

Farmacopéia Brasileira. $4^{\mathrm{a}}$ Ed. São Paulo: Atheneu, 1988. Parte 1.

FERREIRA, A. O. Gotas x Conta-Gotas. Artigos Técnicos, Dicas Farmacotécnicas, 2007. Disponível em : www.ortofarma.com.br/INTRANET/Web\%20Forms/arquivos/ Artigos\%20t\%C3\%A9cnicos/2008/Gotas.pdf Acesso em: 18 de maio de 2009.

GOODMAN, Alfred Gilman. As bases farmacológicas da terapêutica. $10^{\mathrm{a}}$ ed. Rio de Janeiro: McGraw-Hill, 2003.

GUIMARÃES, Hélio Penna; BARBoSA, Lílian Mazza; LARANJEIRA, Lígia Nasi; AVEZUM, Álvaro. Estudos de farmacoeconomia e análises econômicas: conceitos básicos. Revista Brasileira de Hipertensão vol.14, n4, p.265-268, 2007.

JOAQUÍN, R.B.; GAMO, M.J.O. Manual para la correcta redaccíon de la prescripcíon médica. La Revista O.F.I.L., v.9, $2^{\mathrm{a}}$ época, n.4,p 23-29,1999.

KOROLKOVAS, Andrejus; FRANÇA, Francisco F. de A. C. Dicionário terapêutico guanabara. $13^{a}$ ed. Rio de Janeiro: Guanabara Koogan, 2006/07.

MOTA, D. M.; FERNANDES, M. E. P.; COELHO, H. L. Farmacoeconomia: um instrumento de eficiência para a Política de Medicamentos do Brasil. Acta Farmacéutica Bonaerense. v. 22, $n^{\circ}$ 2, 2003. p. 117-186. 
PRATA Júnior, J.A.; PRATA, J.A. Comparação do volume da gota e custo do tratamento dos genéricos de maleato de timolol $0,5 \%$. Arquivos Brasileiros de Oftalmologia. 2004;67(3):419-22.

ROIZENBLATT, R.; FREITAS, D. BELFORT, R. JR.; HOFLING-LIMA, A.L.; PRATA JR, J.A. Impacto econômico no tratamento do glaucoma: volume de gotas de colírios antiglaucomatosos brasileiros e norte-americanos. Arquivos Brasileiros de Oftalmologia. 2001; 64: 143-6.

SECOLI, Silvia Regina; PADILHA, Kátia Grillo; LITVOC, Júlio and MAEDA, Sayuri Tanaka. Farmacoeconomia: perspectiva emergente no processo de tomada de decisão. Ciências \& Saúde Coletiva . 2005, vol.10, suppl., pp. 287-296.

STEINHORST, Alcantara G. K.; VIRTUOSO, S.; MENEGATTI, Oliveira S. M. Avaliação dos Frascos Conta-Gotas de Diferentes Marcas de Diclofenaco Resinato Comercializados no Mercado Brasileiro. Visão Acadêmica, Curitiba, v.8, n.2, Jul. - Dez./2007.

STILLITANO, I.G.; TENÓRIO, A.; CARDOSO, G.; RIBEIRO, M.P.; FIGUEIROA, J.N. Custo do tratamento de drogas antiglaucomatosas: iatanoprost, travoprost, bimatoprost, unoprostona isopropílica. Arquivos Brasileiros de Oftalmologia. 2003; 66(6):85964. 